\title{
PERCEPÇÃO DOS ESTILOS EDUCATIVOS PARENTAIS E AJUSTAMENTO PSICOLÓGICO DO ADULTO - COMPARAÇÃO ENTRE INDIVÍDUOS COM E SEM PERTURBAÇÕES DEPRESSIVAS ${ }^{1}$
}

\author{
Anabela Fernandes Araújo ${ }^{2}$ \\ Hospitais da Universidade de Coimbra - Portugal
}

\begin{abstract}
RESUMO: O tema das possíveis relações entre as práticas educativas parentais, o desenvolvimento de traços de personalidade e a emergência de perturbações psicopatológicas tem sido alvo de grande interesse por parte de investigadores e clínicos; porém, muitos aspectos acerca das suas possíveis consequências ainda não são suficientemente conhecidos. Neste estudo comparam-se duas amostras - adultos com e sem diagnóstico de depressão - em termos de suas memórias das práticas educativas dos pais na infância e adolescência. Apesar de se tratar de um estudo exploratório, os resultados obtidos indicam uma relação significativa entre determinadas atitudes dos pais (sobreprotecção, rejeição e suporte emocional) e o posterior ajustamento ou perturbação emocional do adulto.
\end{abstract}

Palavras-Chave: Estilos educativos parentais; ajustamento psicológico.

\section{THE PERCEPTION OF PARENTS' EDUCATIONAL STYLE AND ADULTS PSYCHOLOGICAL ADJUSTMENT - A COMPARISON BETWEEN PEOPLE WITH AND WITHOUT DEPRESSIVE DISTURBANCES}

\begin{abstract}
The issue of possible relationships between perceived parental rearing styles, the development of personality characteristics and psychopathological disorders is a topic of great interest, as many of its aspects remain insufficiently known. In this study we've compared two samples - depressed versus non-depressed adult subjects - assessing their memories of parental rearing styles in childhood and adolescence. Although this is an exploratory research, the results obtained indicate a significant relationship between a number of parental attitudes (overprotection, rejection and emotional support) and adult adjustment or disturbance.
\end{abstract}

Key-Word: Parental educational styles; psychological adjustment.

A formação das relações interpessoais é um dos aspectos mais significativos do desenvolvimento humano; porém, o conhecimento desses processos e dos mecanismos subjacentes ainda constitui um desafio à investigação, despertando o interesse de autores de diversas orientações teóricas e metodológicas (Gittleman, Klein, Smider \& Essex, 1998; Perris, 1994; Waters \& Cummings, 2000). Tem sido prestada particular atenção ao estudo de possíveis ligações entre as práticas parentais e características de personalidade ou níveis de psicopatologia nos filhos, embora nem sempre com o rigor metodológico e a

\footnotetext{
' Artigo recebido para publicação em 03/2002; aceito em 09/2002

2 Endereço para correspondência: Anabela Fernandes Araújo, Consulta de Acompanhamento Psicologico, Maternidade Dr. Daniel de Matos, Rua Miguel Torga, 3000, Coimbra, Portugal. Email: af_araujo@hotmail.com
}

abrangência necessários (Perris, 1994; Van de Vijver \& Poortinga, 1994).

$\mathrm{O}$ pressuposto de que as práticas educativas parentais podem exercer um impacto profundo no desenvolvimento psicossocial da criança vem sendo formulado desde há muito por diversos autores. Porém, este longo passado acaba por corresponder a uma curta história. O interesse sistemático pelas possíveis consequiências das práticas parentais sobre os descendentes tem início no século XX, sendo nítido tanto no desenvolvimento manifesto nas teorias psicanalíticas (Freud, 1972; Schaffer, 1996), quanto na ênfase colocada nos processos de aprendizagem pelas correntes behavioristas (Perris, 1994; Watson, 1928, 1929, 1930, apud Schultz \& Schultz, 2000) e pela Teoria da Aprendizagem Social (Bandura, 1986) 


\section{Anabela Fernandes Araújo}

e ainda nas múltiplas evidências, que a investigação e prática clínicas têm permitido acumular (Canavarro, 1999; Gittleman, Klein, Smider \& Essex, 1998; Hinde \& Stevenson-Hinde, 1986; Perris, 1994).

Ao enfatizar as relações afetivas estabelecidas com os pais como determinantes do desenvolvimento psicossocial e psicopatológico posterior do sujeito não se pretende a procura improfícua de uma ligação de tipo causa-efeito. Ao invés, torna-se necessária a adoção de uma visão dinâmica, considerando a criança como participante ativa do seu desenvolvimento e, no sentido do conceito de determinismo recíproco formulado por Bandura (1977), salientando a influência que ela exerce sobre as atitudes e comportamentos dos progenitores. A complexidade das interações em jogo é corroborada pelos resultados de estudos que apontam para o impacto intergeracional e do meio social (Belsky \& Isabella, 1988; Perris, 1994).

Ao reconhecer que o desenvolvimento psicossocial é largamente influenciado pela qualidade das relações experienciadas (Amato \& Gilbreth, 1999; Amini \& Cols., 1996; Chen \& Kaplan, 2001; Waters \& Cummings, 2000), é importante compreender como estas se formam, mantêm e modificam, conhecer as suas características e dinâmica intrínseca, entendendo que estas são indissociáveis da personalidade dos sujeitos e do contexto social em que ocorrem (Hinde \& Stevenson-Hinde, 1986; Richters \& Waters, 1991).

O potencial impacto das relações afetivas, vividas ao longo da infância e adolescência no contexto das interações com pais, no posterior ajustamento e saúde mental do adulto, tem sido repetidamente abordado e discutido. Contribuições fundamentais para este domínio foram trazidos pela Teoria do Apego, expressas sobretudo nos trabalhos de Bowlby (1969,
1973, 1980, 1985), Ainsworth $(1969,1989)$.

Muitas das teorias que, historicamente, associaram relações afetivas e psicopatologia, foram elaboradas com base em premissas diferentes das atualmente defendidas. De acordo com Canavarro (1999), as primeiras abordagens concebiam separadamente a infância e a vida adulta, não tendo subjacente uma visão integradora do desenvolvimento. Nos adultos, as ligações entre relações afetivas na infância e posterior ajustamento emocional centravam-se sobre os estilos parentais, e não sublinhavam os aspectos transacionais da relação; as teses defendidas apenas contemplavam o desenvolvimento patológico. Embora não se inclua no âmbito do nosso estudo a exploração da influência das relações afetivas estabelecidas na idade adulta sobre a saúde mental, as perspectivas que o enquadram trazem uma visão desenvolvimental que prossegue ao longo do ciclo de vida, integrando as diversas e sucessivas experiências e significações do indivíduo.

Resultados apoiam a hipótese de que as experiências relacionais precoces, nomeadamente as ligadas com as práticas educativas dos pais, influenciam as estruturas cognitivas responsáveis pelas representações das relações afetivas em fases posteriores do ciclo de vida (Canavarro, 1999; Crook, Raskin \& Elliot, 1981; para uma revisão, Richter, Eisemann \& Perris, 1994).

A conjugação de variáveis culturais, biológicas e psicossociais influencia quer o desenvolvimento da vulnerabilidade do indivíduo para a manifestação de psicopatologia, quer sua interação com o meio (Perris, 1994). Esta abordagem encontra-se esquematizada na figura 1

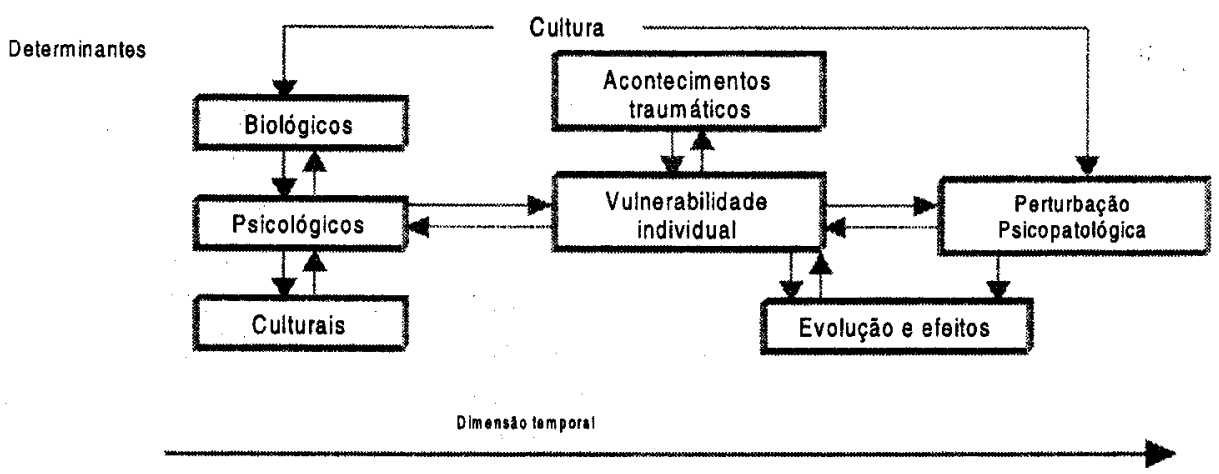

Figura 1:Modelo geral da ocorrência de psicopatologia e sua evolução, centrado no conceito de vulnerabilidade individual (Perris, 1994, p. 15) 
A interação de variáveis leva a que a vulnerabilidade individual não seja vista como um traço estático, mas concebida como condição epigênica, que envolve transações entre a pessoa e o meio ao longo de todo o ciclo de vida. Nesta perspectiva, o papel dos acontecimentos pode aumentar a vulnerabilidade de cada um, e mesmo conduzir à descompensação emocional, ou representar uma forma de proteção contra adversidades futuras.

Da revisão da literatura acerca das relações com os pais na infância e adolescência e saúde mental (Araújo, 2001), é possível concluir que estas se encontram ligadas ao ajustamento psicológico e saúde mental dos indivíduos na idade adulta.

As investigações mostram, de forma consistente, que relações caracterizadas por insegurança, pouca proximidade emocional e estilos educativos parentais marcados por falta de apoio, interesse e carinho, se associam a perturbações psicopatológicas na idade adulta. Na sua maioria, os estudos apontam um efeito maior por parte das relações estabelecidas com a mãe, por contraste àquelas com o pai, no mesmo período de tempo. Os resultados são mais contraditórios no que concerne às práticas de superproteção e rejeição. Também a importância das relações com o progenitor do mesmo sexo para a saúde mental, particularmente no caso mãe-filha, é menos consistente e mais variável em termos de dados empíricos encontrados (Burbach \& Borduin, 1986; Canavarro, 1999; Crook, Raskin \& Elliot, 1981; Eisemann, 1988).

Alguns estudos isolados procuraram conhecer as influências de variáveis como a saúde mental dos progenitores, idade de aparecimento da perturbação e grupo sócio-econômico sobre os estilos educativos parentais. Os resultados indicam que a presença de psicopatologia num ou em ambos os progenitores e um início mais precoce da sintomatologia se ligam à percepção de estilos educativos parentais mais negativos e disfuncionais. O efeito do grupo social de pertença só se faz sentir nos estilos parentais de rejeição e superproteção, sendo mais significativo nas classes mais baixas (Arrindell e Cols., 1986; Eisemann, \&, Perris \& vonKnorring, 1984; Perris, Perris \& Eisemann, 1987).
Refira-se ainda que algumas investigações apresentam resultados em que diferentes estilos de relacionamento correspondem a tipos distintos de psicopatologia na idade adulta. Ora, na maioria dos casos, estes resultados só adquirem significado quando se comparam grupos de doentes com outros da população geral, diminuindo os efeitos quando a comparação é entre grupos com diferentes patologias. Assim, a maior parte das investigações conclui a favor de um efeito não específico das relações afetivas estabelecidas na infância e adolescência sobre a saúde mental e psicopatologia (Perris, Arrindell \& Eisemann, 1994).

\section{Objetivo}

O principal objetivo do presente trabalho foi o estudo das possíveis relações entre os estilos educativos parentais na infância e adolescência, tal como são percebidos posteriormente pelo indivíduo, e o ajustamento psicológico na vida adulta, considerando como indicadores deste a existência ou ausência de perturbações depressivas, definidas segundo a classificação do DSM-IV - Diagnostic and Statistical Manual of Mental Disorders (APA, 1994).

\section{Método}

\section{Amostra}

A amostra foi constituída por indivíduos que procuraram ajuda nos serviços de Psiquiatria e Psicologia dos Hospitais da Universidade de Coimbra, Portugal, tendo sido diagnosticados com Perturbação Depressiva ${ }^{3}$ (Grupo das Perturbações Depressivas - GPD), e por indivíduos da população geral (Grupo Controle - GC), habitantes na zona central de Portugal (distritos de Coimbra, Viseu e Aveiro); o número de homens e mulheres é igual, na sua maioria casados, com grau de instrução secundário ou superior (GC) e básico ou superior (GPD). Os sujeitos do GPD foram abordados na fase final do tratamento, ou já no follow-up, portanto em momento de remissão da sintomatologia.

Ambos os grupos eram equivalentes na sua

\footnotetext{
${ }^{3}$ O diagnóstico foi confirmado no processo clínico dos doentes e pelo médico ou psicoterapeuta assistente.
} 


\section{Anabela Fernandes Araújo}

distribuição em relação à idade $(t=-.152$, g.l. $=198$, $p=.879$ ), e eram também equivalentes em relação ao estado civil $\left(\chi^{2}=7.062\right.$, g.l. $\left.=3, p=.070\right)$, grau de instrução $\left(\chi^{2}=.054\right.$, g.l. $\left.=3, p=.997\right)$ e camada sócioeconômica $\left(\chi^{2}=5.634\right.$, g.l. $\left.=3, p=.131\right)$.
Uma análise mais detalhada da composição dos grupos, tendo em conta a sua distribuição por sexo, estado civil, grau de instrução, camada social e idade encontra-se no quadro 1.

Quadro 1: Características gerais da amostra.

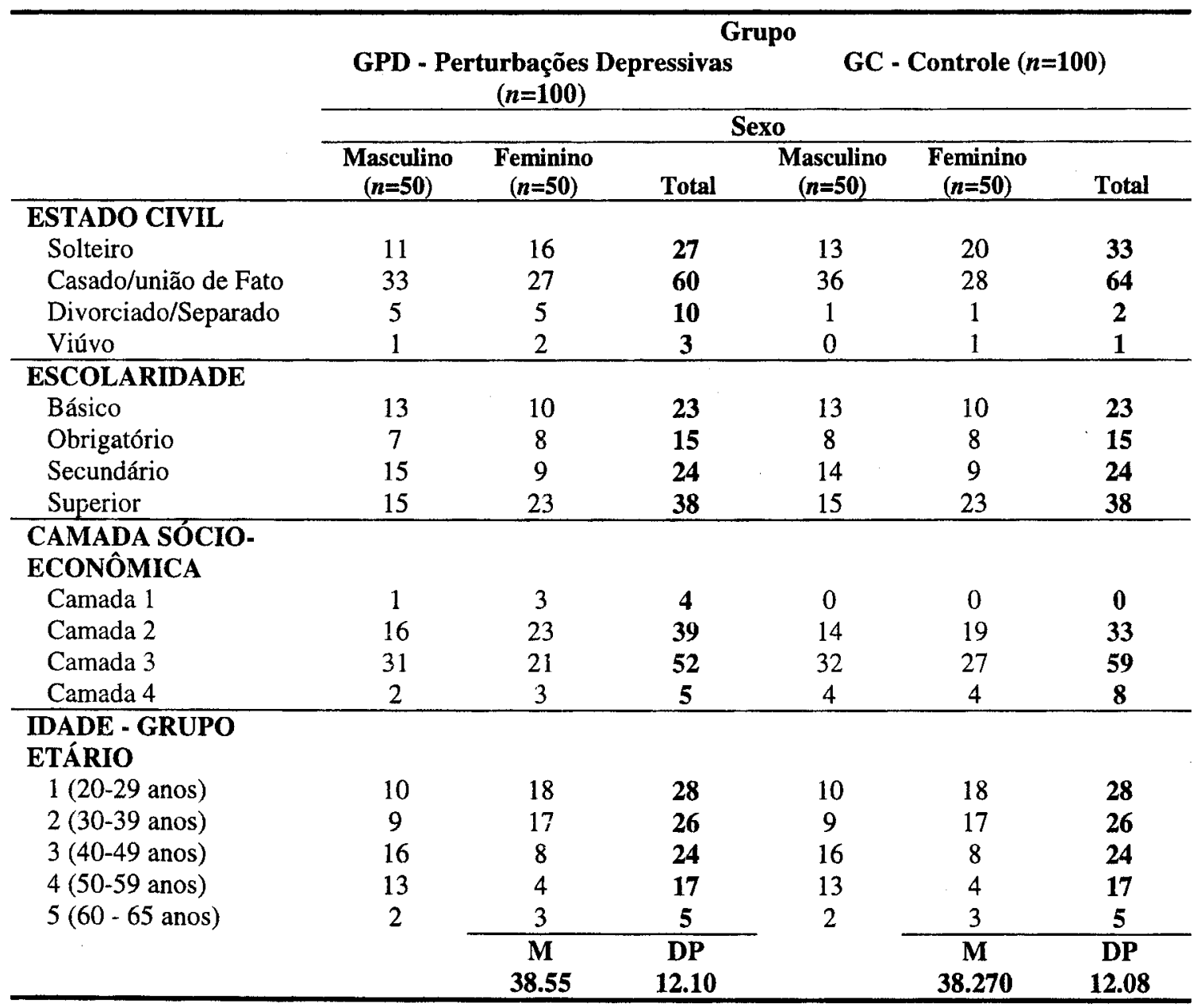

\section{Instrumentos}

- EMBU - Eggna Minnen Beträffande Uppfostran (Memórias de Infância). Atualmente é um dos instrumentos mais utilizados para avaliar a percepção dos adultos acerca das práticas educativas dos seus progenitores (Rapee, 1997). A primeira versão do EMBU foi construída e desenvolvida na Suécia, em 1980, por C. Perris, L. Jacobson, H. Lindstrom, L. von Knorring e H. Perris (1980); a validação científica para Portugal foi realizada por Canavarro (1999). Esta escala procura medir a frequiência da ocorrência de determinadas práticas educativas durante a infância $\mathrm{e}$ adolescência do indivíduo, em relação ao pai e à mãe separadamente. Essa ocorrência é registrada segundo uma escala de tipo Likert de quatro pontos, que vai desde "Não, nunca" até "Sim, a maior parte do tempo". A versão portuguesa é constituída por 23 itens, que submetidos a uma análise fatorial 
permitiram a extração de três fatores, para o pai e a mãe: Apoio Emocional, Rejeição e Superproteção (Canavarro, 1999).

\section{- IACLIDE - Inventário de Avaliação da} Depressão. Foi criado e desenvolvido por Vaz Serra (1994) uma escala de autoavaliação, de tipo Likert, para medir a intensidade dos quadros depressivos, sendo constituído por 23 questões, que se referem a quatro tipos distintos de sintomas presentes nos quadros das Perturbações Depressivas: biológicas, cognitivas, interpessoais e de desempenho de tarefa. Segundo Vaz Serra (1994), "significam a relação que o sujeito deprimido estabelece com o corpo, consigo próprio, com os outros e com o trabalho" (p. 11). Apresenta boas características psicométricas. Neste estudo, o instrumento foi utilizado para obter mais um dado confirmatório do estado depressivo dos sujeitos, para além das avaliações clínicas. Os resultados obtidos serviram de critério de exclusão da amostra.

\section{Procedimento}

Os sujeitos que fazem parte da amostra foram avaliados individualmente através de um conjunto de questionários e de breve entrevista estruturada para coleta de variáveis sócio-demográficas, utilizando sempre os mesmos procedimentos. Em primeiro lugar, era dirigido ao indivíduo um pedido de colaboração voluntária, sendo-lhe fornecidas explicações acerca da natureza do estudo e do tipo de tratamento dos dados, e garantindo a total confidencialidade das respostas. Em seguida, obtido o seu consentimento, procedia-se ao preenchimento dos questionários, com instruções padronizadas.

Alguns dos casos recolhidos foram excluídos da amostra, em conformidade com os seguintes critérios: do ponto de vista do diagnóstico, ocorrência concomitante de Perturbações Psicóticas, Perturbações Bipolares, Perturbações de Personalidade e Perturbações Relacionadas com Substâncias; dificuldades recorrentes na compreensão dos questionários utilizados, e obtenção de uma pontuação superior a 53 no IACLIDE (nesta escala, valores superiores ao indicado indiciam um grau de depressão grave). Efetuada esta seleção o GPD integrou um total de 100 sujeitos.

Na constituição do GC foram excluídos, numa primeira fase, os indivíduos que responderam positivamente à questão Atualmente está a fazer tratamento psiquiátrico ou psicológico?, ou que referiram sofrer, ou ter sofrido dos nervos no passado. Porém, optando por um critério mais cauteloso, numa fase seguinte excluíram-se também todos os que obtiveram uma pontuação superior a 20 no IACLIDE pois, de acordo com o autor da escala, o grau de depressão leve situar-se-á entre os pontos 20 e 34 (Vaz Serra, 1994), pelo que se poderá considerar um resultado inferior a 20 indicador de ausência de depressão.

Excluíram-se também todos aqueles que na infância e adolescência não habitaram continuamente com ambos os progenitores - a perda ou separação de um ou ambos os progenitores é um fator considerado relevante no aumento da vulnerabilidade dos indivíduos e na posterior manifestação de perturbações depressivas (Ainsworth, 1969, 1989; Bowlby, 1973; Lewinsohn \& Rosenbaum, 1987; Perris, Maaj, Perris \& Eisemann, 1985; Perris \& Cols., 1986; Richter, 1994; Saranson, Pierce, Shearin, Saranson \& Waltz, 1991), pelo que pretendemos deste modo controlar o efeito de uma variável que não era objeto deste trabalho.

\section{Resultados}

\section{Estilos educativos parentais}

Começamos por estudar as diferenças entre o GPD (grupo das Perturbações Depressivas) e o GC (grupo controle) relativamente à representação dos estilos educativos dos pais avaliados através do EMBU. Fizemo-lo separadamente para o pai e para a mãe, através de testes-t, cujos resultados se encontram no quadro 2 . 


\section{Anabela Fernandes Araújo}

Quadro 2: Comparação dos grupos em relação aos fatores do EMBU.

\begin{tabular}{|c|c|c|c|c|c|c|}
\hline & \multicolumn{2}{|c|}{ GPD } & \multicolumn{2}{|c|}{ GC } & \multirow[b]{2}{*}{ teste-t } & \multirow[b]{2}{*}{$\mathbf{p}$} \\
\hline & Média & DP & Média & DP & & \\
\hline \multicolumn{7}{|l|}{$\overline{\text { Pai }}$} \\
\hline Apoio emocional & 14.750 & 4.916 & 18.840 & 5.510 & 5.539 & n.s. \\
\hline Rejeição & 13.860 & 5.224 & 11.170 & 4.035 & -4.075 & .000 \\
\hline Superproteção & 15.060 & 3.640 & 13.560 & 2.983 & -3.188 & .002 \\
\hline \multicolumn{7}{|l|}{ Mãe } \\
\hline Apoio emocional & 15.890 & 5.097 & 20.920 & 4.836 & 7.159 & .000 \\
\hline Rejeição & 17.150 & 6.423 & 12.810 & 3.637 & -5.880 & .000 \\
\hline Superproteção & 17.560 & 4.081 & 14.190 & 2.740 & -6.856 & .000 \\
\hline
\end{tabular}

Quando comparado com o GC, o GPD percepciona as suas infância e adolescência como pontuadas por menor apoio emocional e maior rejeição e superproteção por parte de ambos os progenitores; as diferenças são significativas entre os dois grupos em relação a todos os fatores, exceto à dimensão de apoio emocional por parte do pai.

Em todas as dimensões consideradas, foi possível observar que as médias representativas dos estilos educativos da mãe eram superiores às do pai. Os resultados confirmaram que as diferenças eram significativas para as três dimensões, o que permite concluir que os estilos educativos maternos são percepcionados pelos indivíduos como mais intensos que os paternos.

Efetuamos também análises comparativas diferenciando os inđivíduos não apenas pelos grupos definidos em função da presença ou ausência de Perturbações Depressivas, mas ainda em função do sexo. Os resultados patentes nos quadros 3 e 4 permitem verificar que não há diferenças significativas nos estilos educativos parentais percepcionados por homens e mulheres.

Quadro 3: Comparação dos estilos educativos parentais em função do sexo Grupo das Perturbações Depressivas (GPD)

\begin{tabular}{|c|c|c|c|c|c|c|}
\hline & \multicolumn{2}{|c|}{ Homens } & \multicolumn{2}{|c|}{ Mulheres } & \multirow[b]{2}{*}{ teste-t } & \multirow[b]{2}{*}{$\mathbf{p}$} \\
\hline & Média & $\mathbf{D P}$ & Média & DP & & \\
\hline \multicolumn{7}{|l|}{ Pai } \\
\hline Apoio emocional & 13.820 & 4.750 & 15.680 & 4.950 & -1.917 & n.s. \\
\hline Rejeição & 14.280 & 5.240 & 14.280 & 5.230 & .802 & n.s. \\
\hline Superproteção & 14.900 & 3.600 & 15.220 & 3.710 & -.438 & n.s. \\
\hline \multicolumn{7}{|l|}{ Mãe } \\
\hline Apoio emocional & 16.120 & 5.290 & 15.660 & 4.930 & .449 & n.s. \\
\hline Rejeição & 16.120 & 6.360 & 18.180 & 6.380 & -1.617 & n.s: \\
\hline Superproteção & 16.880 & 4.320 & 18.240 & 3.750 & -1.682 & n.s. \\
\hline
\end{tabular}


Quadro 4: Comparação dos estilos educativos parentais em função do sexo Grupo Controle (GC).

\begin{tabular}{lccllllll}
\hline & \multicolumn{2}{c}{ Homens } & & \multicolumn{2}{c}{ Mulheres } & & \\
\cline { 2 - 3 } & Média & DP & & Média & DP & teste-t & p \\
\hline Pai & & & & & & & \\
$\quad$ Apoio emocional & 18.180 & 5.060 & & 19.500 & 5.900 & -1.201 & n.s. \\
$\quad$ Rejeição & 11.560 & 3.910 & & 10.780 & 4.160 & .966 & n.s. \\
$\quad$ Superproteção & 14.120 & 2.900 & & 13.000 & 2.990 & 1.902 & n.s. \\
& & & & & & \\
Mãe & & & & & & \\
$\quad$ Apoio emocional & 20.360 & 4.890 & & 21.480 & 4.770 & -1.160 & n.s. \\
$\quad$ Rejeição & 12.860 & 3.430 & & 12.760 & 3.870 & .137 & n.s. \\
$\quad$ Superproteção & 14.520 & 2.750 & & 13.860 & 2.720 & 1.207 & n.s. \\
\hline
\end{tabular}

É possível observar que as mulheres do GPD percepcionam um nível mais baixo de apoio emocional e níveis mais elevados de rejeição e superproteção relativamente aos estilos educativos maternos, por comparação com o percebido pelos indivíduos do sexo masculino (quadro 3). Nas mulheres do GC acontece o contrário (quadro 4).

Efeitos dos estilos educativos parentais percepcionados na infância e adolescência sobre a saúde mental do adulto
Com o intuito de confirmar e explorar mais extensivamente os dados obtidos tentamos em seguida determinar quais as variáveis independentes que melhor diferenciavam o GPD do GC, permitindo uma classificação mais correta nos grupos estabelecidos. Para a prossecução deste objetivo utilizamos a análise de função discriminante de modelo completo.

Nos quadros 5 e 6 registram-se os resultados da análise de função discriminante efetuada.

Quadro 5: Análise da função discriminante entre GPD vs. GC.

\begin{tabular}{lcl}
\hline Variável & $\boldsymbol{F}(\mathbf{1 , 1 9 8})$ & $p$ \\
\hline Estilos educativos & & \\
$\quad$ Apoio emocional (pai) & 30.678 & .000 \\
Rejeição (pai) & 16.605 & .000 \\
Superproteção (pai) & 10.161 & .002 \\
& & \\
Apoio emocional (mãe) & 51.253 & .000 \\
Rejeição (mãe) & 34.569 & .000 \\
Superproteção (mãe) & 47.003 & .000 \\
\hline Lambda de Wilks $=.681$ & $F=15.081$ & \\
g.1.=6 & $p=.000$ & \\
\hline
\end{tabular}

Colocaram-se como variáveis independentes ou preditoras, no modelo discriminante, as dimensões dos estilos educativos parentais, separadamente para o pai e a mãe, pois os dados anteriormente obtidos eram indicativos de que a representação das práticas educativas maternas e paternas poderia exercer efeitos explicativos das diferenças entre os grupos.

A variável dependente era a existência ou não de Perturbações Depressivas no indivíduo.
Os resultados mostram, como se pode verificar no quadro 5 , que a análise de função discriminante é estatisticamente significativa como um todo, existindo diferenças multivariadas entre os dois grupos $(\mathrm{F}(1,198)=15.081, p=.000)$. O valor do lambda de Wilks revela que a percentagem de variância dos valores discriminantes explicada pela pertença ao grupo é de $32 \% 4$.

Averiguamos a importância das variáveis para 
a diferenciação dos grupos através dos valores do $F$ univariado e da significância estatística, verificando que todas contribuem significativamente para a diferenciação entre eles.

No quadro 5 podemos observar que o maior contributo para a diferença entre os grupos é dado pela representação das práticas educativas de apoio emocional da mãe. As variáveis relacionadas com os estilos educativos maternos, são, de resto, as que mais contribuem para a distinção dos grupos. Os valores das médias das variáveis preditoras (quadro 2) permitem verificar em que sentido ocorrem as dife- renças entre os grupos. Assim, os indivíduos pertencentes ao GPD, por comparação com o GC, percepcionam que os estilos educativos dos progenitores foram pautados por menor apoio emocional e maior superproteção e rejeição; as diferenças são mais acentuadas relativamente às práticas educativas maternas.

A análise de função discriminante foi também utilizada com o objetivo de avaliar a adequação da classificação dos sujeitos. O conjunto das variáveis permitiu uma classificação correta de $74 \%$ dos indivíduos, com $74 \%$ de acertos no GPD e $73 \%$ no GC (quadro 6).

Quadro 6: Análise da função discriminante entre GPD vs. GC: eficácia da classificação.

\begin{tabular}{|c|c|c|c|}
\hline & \multicolumn{2}{|c|}{ Grupo predito } & \multirow[b]{2}{*}{$\begin{array}{c}\text { \% classificação } \\
\text { correta }\end{array}$} \\
\hline & $\begin{array}{c}\text { Grupo de } \\
\text { Controlo (GC) } \\
\end{array}$ & $\begin{array}{c}\text { Perturbações } \\
\text { Depressivas (GPD) }\end{array}$ & \\
\hline \multicolumn{4}{|l|}{ Grupo observado } \\
\hline Grupo Controle (GC) & 73 & 27 & 73 \\
\hline Perturbações Depressivas(GPD) & 26 & 74 & 74 \\
\hline Total & 99 & 101 & 74 \\
\hline
\end{tabular}

A porcentagem de classificações corretas obtidas pela função discriminante, quando comparada com a percentagem de classificações certas que se obteriam com base na sorte ou acaso, indica-nos que a função aumenta consideravelmente a capacidade de predizer corretamente os controles e os indivíduos com Perturbação Depressiva ${ }^{5}$.

\section{Efeitos dos estilos educativos parentais percepcionados na infância e adolescência sobre a saúde mental do adulto - diferenças em função do gênero dos indivíduos}

Com o objetivo de determinar se as dimensões dos estilos educativos parentais, que assumiam maior relevância para a discriminação dos grupos controles versus indivíduos com perturbação emocional, eram as mesmas para os homens e as mulhe-

\footnotetext{
4 O lambda de Wilks corresponde à porcentagem da variância dos valores discriminantes, não explicada pela pertença ao grupo. Varia entre $0 \mathrm{e} 1$, pelo que ao subtrair-se-lhe a unidade obtém-se a percentagem de variância explicada pela pertença ao grupo. Este valor reflete também a importância de cada varível, tal como os valores do $F$ e $p$.
}

res, procedemos a subseqüentes análises de função discriminante utilizando as mesmas variáveis independentes, mas separando os indivíduos em função do gênero, masculino ou feminino.

Obtivemos assim quatro grupos, cada um com 50 sujeitos (homens com e sem perturbação depressiva, mulheres com e sem perturbação depressiva).

Os homens do GPD quando comparados com os do GC, percepcionam os estilos educativos parentais como caracterizados por menos práticas de apoio social e mais práticas de superproteção e rejeição. A falta de apoio emocional, sobretudo do pai, parece ser particularmente importante para distinguir o GPD do GC.

O conjunto das variáveis permite uma classificação correta de $71 \%$, com $74 \%$ de acertos no GPD e $68 \%$ no GC, como se pode verificar no quadro seguinte.

\footnotetext{
${ }^{5}$ Aumenta de $50 \%$ para $74 \%$ a porcentagem de classificações corretas.
} 
Quadro7: - Análise da função discriminante entre indivíduos do sexo masculino do GPD vs. GC.

\begin{tabular}{lcc}
\hline \multicolumn{1}{c}{ Variável } & $\boldsymbol{F}(\mathbf{1 , 9 8 )}$ & $\boldsymbol{p}$ \\
\hline Estilos educativos & & \\
$\quad$ Apoio emocional (pai) & 19.762 & .000 \\
Rejeição (pai) & 8.655 & .004 \\
Superproteção (pai) & 1.425 & .235 \\
& & \\
Apoio emocional (mãe) & 17.322 & .002 \\
Rejeição (mãe) & 10.182 & .000 \\
Superproteção (mãe) & 10.629 & .002 \\
\hline Lambda de Wilks=.781 & $F=6.074$ \\
g.l. $=6$ & $p=.000$ \\
\hline
\end{tabular}

Quadro 8: Análise de função discriminante entre indivíduos do sexo masculino do GPD vs. GC - eficácia da classificação.

\begin{tabular}{lccc}
\hline & \multicolumn{2}{c}{ Grupo predito } & \% classificação \\
\cline { 3 - 4 } & $\begin{array}{c}\text { Grupo Controle } \\
\text { (GC) }\end{array}$ & $\begin{array}{c}\text { Perturbações } \\
\text { Depressivas (GPD) }\end{array}$ & $\begin{array}{c}\text { correta } \\
\text { Grupo observado }\end{array}$ \\
\hline & 34 & & 68 \\
Grupo Controle (GC) & 13 & 16 & 74 \\
Perturbações Depressivas (GPD) & & 37 & $\mathbf{7 1}$ \\
Total & 47 & 53 & \\
\hline
\end{tabular}

Os resultados obtidos para as mulheres do GPD comparado com o GC constam dos quadros 9 e 10. Mais uma vez os resultados mostram que a análise de função discriminante é significativa como um todo, apontando para a existência de diferenças significativas entre os grupos.

Quadro 9: Análise da função discriminante entre indivíduos do sexo feminino do GPD vs. GC.

\begin{tabular}{lcl}
\hline \multicolumn{1}{c}{ Variável } & $\boldsymbol{F ( 1 , 9 8 )}$ & $\boldsymbol{p}$ \\
\hline Estilos educativos & 12.280 & .001 \\
Apoio emocional (pai) & 7.927 & .006 \\
Rejeição (pai) & 10.853 & .001 \\
Superproteção (pai) & & \\
& 35.961 & .000 \\
Apoio emocional (mãe) & 26.359 & .000 \\
Rejeição (mãe) & 44.715 & .000 \\
Superproteção (mãe) & $F=10.649$ & \\
\hline Lambđa de Wilks= .593 & $p=.000$ \\
g.l.=6 &...
\end{tabular}

representativas das práticas educativas da mãe as mais importantes na distinção de mulheres com e sem perturbação depressiva. Atendendo às médias obtidas e atrás referidas, as mulheres do GPD percepcionam por maiores índices de superproteção e rejeição e menores índices de apoio emocional do que as do GC.

O quadro 10 mostra-nos a eficácia da classificação dos grupos em função da análise realizada. 
Quadro 10: Análise de função discriminante entre indivíduos do sexo feminino do GPD vs. GC - eficácia da classificação.

\begin{tabular}{|c|c|c|c|}
\hline & \multicolumn{2}{|c|}{ Grupo predito } & \multirow[b]{2}{*}{$\begin{array}{l}\text { \% classificação } \\
\text { correta }\end{array}$} \\
\hline & $\begin{array}{l}\text { Grupo Controle } \\
\text { (GC) }\end{array}$ & $\begin{array}{c}\text { Perturbações } \\
\text { Depressivas (GPD) }\end{array}$ & \\
\hline \multicolumn{4}{|l|}{ Grupo observado } \\
\hline Grupo de Controlo (GC) & 35 & 15 & 70 \\
\hline Perturbações Depressivas (GPD) & 15 & 36 & 72 \\
\hline Total & 49 & 51 & 71 \\
\hline
\end{tabular}

Tal como no grupo de indivíduos do sexo masculino, os sujeitos corretamente classificados são $71 \%$, com $72 \%$ de acertos no GPD e $70 \%$ de acertos no GC.

Destas análises é possível concluir que as dimensões dos estilos educativos parentais mais importantes e significativos para distinguir indivíduos com e sem perturbação depressiva são distintos no sexo masculino e no feminino.

\section{Discussão e Conclusão}

A análise da influência dos estilos educativos parentais na saúde mental dos filhos, quando adultos, revelou uma associação entre as práticas de ambos os progenitores (caracterizadas por menor apoio emocional), e a depressão no adulto. Os indivíduos do grupo de perturbações depressivas (GPD) parecem ter uma percepção dos comportamentos dos pais como sendo mais disfuncionais do que os indivíduos do grupo controle (GC). Estes resultados estão de acordo com os encontrados por muitos estudos (Burbach \& Borduin, 1986; Canavarro, 1999; Crook, Raskin \& Eliot, 1981; Duggan, Sham, Minne, Lee \& Murray, 1998; Richter, 1994; Richter, Eisemann \& Perris, 1994).

Também à semelhança dos resultados de Canavarro (1999), Crook, Raskin e Eliot (1981) e Burbach e Borduin (1986), no todo da amostra os estilos educativos maternos são percebidos como mais intensos. As dimensões de falta de apoio emocional e rejeição por parte da mãe são as que melhor discriminam os indivíduos que sofreram uma perturbação depressiva e os que não registraram perturbações emocionais.
Porém, quando se separa a amostra em função do gênero, verificam-se algumas diferenças - as mulheres parecem relatar mais comportamentos disfuncionais por parte da sua mãe. Assim, quando comparados homens do GPD com os do GC, embora a dimensão de apoio emocional continue a ser a que melhor discrimina, trata-se aqui da percepção da sua falta por parte do progenitor do mesmo sexo (resultados concordes com os Richter, 1994); os estilos educativos maternos assumem também um papel relevante.

Ao serem comparadas as mulheres do GPD com as do GC, verifica-se que os estilos educativos maternos são os mais importantes para explicar a diferença entre grupos, mas a dimensão que melhor os discrimina é a superproteção por parte da mãe. De acordo com Crook, Raskin e Eliot (1981), a ligação entre a percepção dos estilos educativos parentais e a depressão pode ser afetada pelo gênero do indivíduo.

Estes resultados parecem apontar para diferentes papéis exercidos pelos progenitores no processo de desenvolvimento dos indivíduos, o que pode ser devido à influência das normas sociais nas práticas tradicionais de socialização, salientando-se a importância das experiências relacionais positivas na infância e adolescência com a mãe, mas também com o pai, enquanto fator significativo para os níveis de ajustamento e bem-estar do adulto.

É importante enfatizar que este estudo não contemplou múltiplas variáveis que poderão contribuir para a complexa teia de interações subjacentes ao ajustamento psicológico e saúde mental do adulto. Desde logo, seria vantajoso estudar os indivíduos, sobretudo os do GPC, num período mais longo de tempo. 
Outras dimensões que reconhecidamente se associam à saúde mental, e que, dadas as características exploratórias deste estudo, não foram avaliadas, ligam-se quer à história de psicopatologia nos pais, quer à estrutura e funcionamento da rede de apoio social, e da adequação das suas trocas, tal como percebida pelos indivíduos; e ainda a qualidade das relações afetivas na idade adulta.

Importaria ainda explorar as diferenças no papel exercido pelos pais ao longo do desenvolvimento, e os fatores que influenciam a construção das representações acerca do comportamento dos de mesmo sexo, e do sexo oposto, uma vez que o impacto dos estilos educativos parentais pareceu, neste estudo, estar associado ao gênero.

Em síntese, os resultados indicam que indivíduos que sofreram no seu percurso de vida uma perturbação depressiva percebem de forma mais negativa as atitudes e comportamentos dos seus progenitores, ao longo da infância e adolescência, do que aqueles que até ao momento do estudo não desenvolveram qualquer perturbação psicopatológica. A magnitude dessas diferenças varia com o sexo do indivíduo e do progenitor, embora as dimensões relacionadas com os estilos educativos maternos sejam, globalmente, preponderantes.

\section{Referências Bibliográficas}

Ainsworth, M.D.S. (1969). Object relations, dependency, and attachment: a theoretical review of the infant-mother relationship. Child development, 40, p. 969-1025.

Ainsworth, M.D.S. (1989). Attachments beyond infancy. American Psychologist, 44, p.709-716.

Amato, P.R.. \& Gilbreth, J.G. (1999). Nonresident fathers and children's well-being: a metaanalysis. Journal of Marriage and the Family, 61 , p. 557-575.

American Psychiatric Association (1994). Diagnostic and statistical manual of mental disorders ( $4^{\text {th }} e d$.). Washington, DC.:Author.

Amini, F., Lewis, T., Lannon, R, Louie, A., et al. (1996). Affect, attachment, memory: contributions toward psychobiologic integration. Psychiatry, 59, p. 213-239.
Antonucci, T.C. (1985). Attachment in adulthood and aging. In M.B. Sperling \& W.H. Berman (Eds.), Attachment in adults - clinical and developmental perspectives (pp. 256-272). New York: Guilford Press.

Antonucci, T.C. \& Akyama, H. (1994). Convoys of attachment and social relations in children, adolescents, and adults. In F. Nestman \& K. Hurrelmann (Eds.), Social networks and social support in childhood and adolescence (pp. 37-52). Berlin: Walter de Gruyter.

Araújo, A.F. (2001). Relações com os pais na infância e adolescência e perturbação emocional no adulto. Dissertação de Mestrado, Universidade de Coimbra, Coimbra - Portugal.

Arrindell, W.A., Perris, C., Eisemann, M., van der Ende \& von Knorring, L. (1986). Cross-national generalizability of patterns of parental rearing styles behaviour: invariance of EMBU dimensional representations of healthy subjects from Australia, Dennmark, Hungary, Italy and The Netherlands. Personality and Individual Differences, 7, p. 103-112.

Bandura, A. (1977). Self-efficacy: toward a unifying theory of behavior change. Psychological Review, 84, p. 191-215.

Bandura, A. (1986). Social foundations of thought and action: a social cognitive theory. Englewood Cliffs: Prentice-Hall.

Belsky, J. \& Isabella, R. (1988). Maternal, infant and social-contextual determinants of attachment security. In J. Belksky \& T. Nezworski (eds.), Clinical implications of attachment (pp. 4194). Hillsdale: Lawrence Earlbaum.

Bowlby, J. (1969). Attachment and Loss I: Attachment..London: Hogarth Press.

Bowlby, J. (1973). Attachment and Loss II: Separation, anxiety and anger. London: Tavistock.

Bowlby, J. (1980). Attachment and Loss III: Loss, sadness and depression. New York: Basic Books. 
Bowlby, J. (1985). The role of childhood experience in cognitive disturbance. In M. Mahoney \& A. Freeman (Eds.), Cognition and psychotherapy (pp. 181-200). New York: Plenum Press.

Burbach, D.J. \& Borduin, C.M. (1986). Parent-child relations and the etiology of depression - a review of methods and findings. Clinical Psychology Review, 6, p.133-153.

Canavarro, M.C. (1999). Relações afectivas ao longo do ciclo de vida e saúde mental. Coimbra: Quarteto.

Chen, Z. \& Kaplan, H.B. (2001). Intergenerational transmission of constructive parenting. Journal of Marriage and the Family, 63, p. 17-31.

Cicchetti, D., Toth, S.L. \& Lynch. M. (1995). Bowlby's dream comes full circle - the application of attachment theory to risk and psychopathology. In T.H. Ollendick \& R.J. Prinz (Eds.), Advances in clinical child psychology (vol. 17, pp. 1-75). New York: Plenum Press.

Collins, N.L. \& Read, S.J. (1994). Cognitive representations of attachment: the structure ans function of working models. In K. Bartholomew \& D. Perlman (Eds.). Attachment processes in adulthood (pp. 53-90). London: Jessica Kingsley Publishers Ltd.

Crook, T., \& Raskin, A. \& Eliot, J. (1981). Parentchild relationships and adult depression. Child Development, 52, p.950-957.

Duggan, C., Sham, P., Minne, C., Lee, A. \& Murray, R. (1998). Quality of parenting and vulnerability to depression: results from a family study. Psychological Medicine, 28, p.185-191.

Eisemann, M. (1988). Praticas educativas de los padres y psicopatologia. Revista de Psiquiatria de Fac. Med. Barna., 15, p.243-254.

Eisemann, M., Perris, C., Perris, H. \& von Knorring, L. (1984). Perceived parental rearing practices in depressed patients in relation to social class. Acta Psychiatrica Scandinavica, 70, p.568572.

Field, T. (1995). Psychological depressed parents. In M.H. Bornstein (Ed.). Handbook of parenting (pp. 85-99). Mahwan: Lawrence Erlbaum Associates.

Freud, S. (1905/1972). Três ensaios sobre a teoria da sexualidade. Lisboa: Livros do Brasil.

Gittleman, M.G., Klein, M.H., Smider, N.A. \& Essex, M.J. (1998). Recollections of parental behaviour, adult attachment and mental health: mediating and moderating effects. Psychological Medicine, 28, p. 1443-1455.

Hazan, C. \& Shaver, P.R. (1987). Romantic love conceptualized as an attachment process. Journal of Personality and Social Psychology, 52, p. 511-524.

Hinde, R.A. \& Stevenson-Hinde, J. (1986). Relating childhood relationships to individual characteristics. In Hartup, W.W. \& Z. Rubin (Eds.), Relationships and development (pp. 2749). Hillsdale: Lawrence Earlbaum Associates.

Lewis, M. (1988). Social development in infancy and early childhood. In J.D. Osofsky (Ed.), Handbook of infant development (pp. 419493). New York: John Wiley and Sons.

Lewinsohn, P.M. \& Rosenbaum, M. (1987). Recall of parental behavior by acute depressives, remitted depressives, and nondepressives. Journal of Personality and Social Psychology, 52, p.611-619.

Perris, C. (1994). Linking the experience of dysfunctional parenting rearing with manifest psychopathology: a theoretical framework. In C. Perris, W.A. Arrindell \& M. Eisemann (Eds.), Parenting and psychopathology (pp. 3-32). Chichester: Wiley.

Perris, C., Arrindell, W.A. \& Eisemann, M. (1994). Parenting and psychopathology. Chichester: Wiley.

Perris, C., Arrindell., W.A., Perris, H., Eisemann, M., van der Ende, J. \& von Knorring, L. (1986). Perceived depriving parental rearing and depression. British Journal of Psychiatry, 148, p. $170-175$.

Perris, C., Jacobsson, L., Lindström, H., von Knorring, L. \& Perris, H. (1980). Development 
of a new inventory for assessing memories of parental rearing behaviour. Acta Psychiatrica Scandinava, 61, p. 265-274.

Perris, C., Maaj, M., Perris, H. \& Eisemann, M. (1985). Perceived parental rearing behaviour in unipolar and bipolar depressed patients. Acta Psychiatrica Scanvdinavica, 72, p.172-175.

Perris, C., Perris, H. \& Eisemann, M. (1987). Perceived parental rearing practices, parental affective disorders and age at onset in depressed patients. International Journal of Family Psychiatry, 8, p.183-199.

Rapee, R.M. (1997). Potential role of childrearing practices in the development of anxiety and depression. Clinical Psychology Review, 17, p.47-67.

Richter, J. (1994). Parental rearing and aspects of psychopathology with special reference to depression. In C. Perris, W.A. Arrindell \& Martin Eisemann (Eds.), Parenting and psychopathology (pp. 235-252). Chichester: Wiley.

Richter, J., Eisemann, M. \& Perris, C. (1994). The raltion between perceived parental rearing and dysfunctional attitudes in unipolar depressive inpatients. Clinical Pscyhology and Psychotherapy, 1, p. 82-86.

Richters, J.E. \& Waters, E. (1991) . Attachment and socialization - the positive side of social influence. In M. Lewis \& S. Feinman (Eds.), Social Influences and socialization in infancy. New York: Plenum.

Rutter, M. (1985). Resilience in the face of adversity: protective factors and resistence to psychiatric disorder. British Journal of Psychiatry, 147, p. 598-611.

Saranson, B.R., Pierce, G.R., Shearin, E.N., Saranson, I.G. \& Waltz, J.A. (1991). Perceived social support and working models of self and actual others. Journal of Personality and Social Psychology, 60, p. 273-287.

Schaffer, H.R. (1996). O desenvolvimento social da criança. Lisboa: Instituto Piaget.
Schultz, D.P. \& Schultz, S.E. (2000). A modern history of psychology. New York: Harcourt College Publishers.

Van de Vijver, F.J.R. \& Poortinga, Y.H. (1994). Methodological issues in cross-cultural studies on parental rearing behavior and psychopathology. In C. Perris, W.A. Arrindell \& Martin Eisemann (Eds.), Parenting and Psychopathology (pp. 173-197). Chichester: Wiley.

Vaz Serra, A. (1994). IACLIDE - Inventário de depressão clínica da depressão. Coimbra: Edição Psiquiatria Clínica.

Waters, E. \& Cummings, E.M. (2000). A secure base from which to explore close relationships. Child relationships. Child Development, special millennium issue. 
228 